\title{
Intra-Aneurysmal Flow Patterns: Illustrative Comparison among Digital Subtraction Angiography, Optical Flow, and Computational Fluid Dynamics
}

O. Brina, R. Ouared, O. Bonnefous, F. van Nijnatten, P. Bouillot, P. Bijlenga, K. Schaller, K.-O. Lovblad, T. Grünhagen, D. Ruijters, and V. Mendes Pereira

\begin{abstract}
BACKGROUND AND PURPOSE: Digital subtraction angiography is the gold standard vascular imaging and it is used for all endovascular treatment of intracranial anerysms. Optical flow imaging has been described as a potential method to evaluate cerebral hemodynamics through DSA. In this study, we aimed to compare the flow patterns measured during angiography, by using an optical flow method, with those measured by using computational fluid dynamics in intracranial aneurysms.
\end{abstract}

MATERIALS AND METHODS: A consecutive series of 21 patients harboring unruptured saccular intracranial aneurysms who underwent diagnostic angiography before treatment was considered. High-frame-rate digital subtraction angiography was performed to obtain an intra-aneurysmal velocity field by following the cardiac-modulated contrast wave through the vascular structures by using optical flow principles. Additionally, computational fluid dynamics modeling was performed for every case by using patient-specific inlet-boundary conditions measured with the optical flow method from both DSA and 3D rotational angiography datasets. Three independent observers compared qualitatively both the inflow direction and the apparent recirculation in regular DSA, optical flow images, and computational fluid dynamics flow patterns for each patient; $\kappa$ statistics were estimated.

RESULTS: We included 21 patients. In 14 of these 21, the flow patterns were conclusive and matching between the optical flow images and computational fluid dynamics within the same projection view $(\kappa=.91)$. However, in only 8 of these 14 patients the optical flow images were conclusive and matching regular DSA images (observer $\kappa=0.87$ ). In 7 of the 21 patients, the flow patterns in the optical flow images were inconclusive, possibly due to improper projection angles.

CONCLUSIONS: The DSA-based optical flow technique was considered qualitatively consistent with computational fluid dynamics outcomes in evaluating intra-aneurysmal inflow direction and apparent recirculation. Moreover, the optical flow technique may provide the premises for new solutions for improving the visibility of flow patterns when contrast motion in DSA is not apparent. This technique is a diagnostic method to evaluate intra-aneurysmal flow patterns and could be used in the future for validation and patient evaluation.

ABBREVIATIONS: $C F D=$ computational fluid dynamics; DVF $=$ detector velocity fields; $O F=$ optical flow

W hile unruptured intracranial aneurysms have become increasingly diagnosed, ${ }^{1}$ more questions have been raised about the improvement of patient care management, requiring the knowledge of risks for both treatment and conservative fol-

Received May 17, 2014; accepted after revision May 19

From the Interventional Neuroradiology Unit (O. Brina, R.O., P. Bouillot, K.-O.L., V.M.P.), Service of Neuroradiology, and Service of Neurosurgery (P. Bijlenga, K.S.), University of Geneva Hospitals and Faculty of Medicine, Geneva, Switzerland; Medisys Research Laboratory (O. Bonnefous) and Interventional X-Ray (F.v.N., T.G., D.R.), Philips Healthcare, Zürich, Switzerland; and Division of Neuroradiology (V.M.P.), Department of Medical Imaging, and Division of Neurosurgery (V.M.P.), Department of Surgery, Toronto Western Hospital, University Health Network, Toronto, Ontario, Canada.

This work was supported by the Swiss National Funds-SNF 32003B_141192.

Please address correspondence to Vitor Mendes Pereira, MD, MSc, Division of Neuroradiology, Department of Medical Imaging, and Division of Neurosurgery, Department of Surgery, Toronto Western Hospital, University Health Network, Toronto, Ontario, Canada; e-mail: vitormpbr@hotmail.com low-up, which still remain unknown. In particular, the role of hemodynamics in predicting both aneurysm rupture and treatment efficacy for flow-diverter stents still remains ambiguous, despite the sustained research effort. ${ }^{2,3}$ Recently, a novel method has been developed based on digital subtraction angiography and $3 \mathrm{D}$ rotational angiography images by using optical flow (OF) principles, permitting the observation and measurement of intracranial hemodynamic characteristics in patients with intracranial aneurysms. Bonnefous et $\mathrm{al}^{4}$ have successfully validated this $\mathrm{OF}$ method for measuring the volumetric flow rate by using in vitro experiments, and Pereira et $\mathrm{al}^{5}$ have validated blood flow rate measurements in patients by using Doppler sonography. This technique has also been applied to quantify flow reduction after

- Indicates open access to non-subscribers at www.ajnr.org

http://dx.doi.org/10.3174/ajnr.A4063 


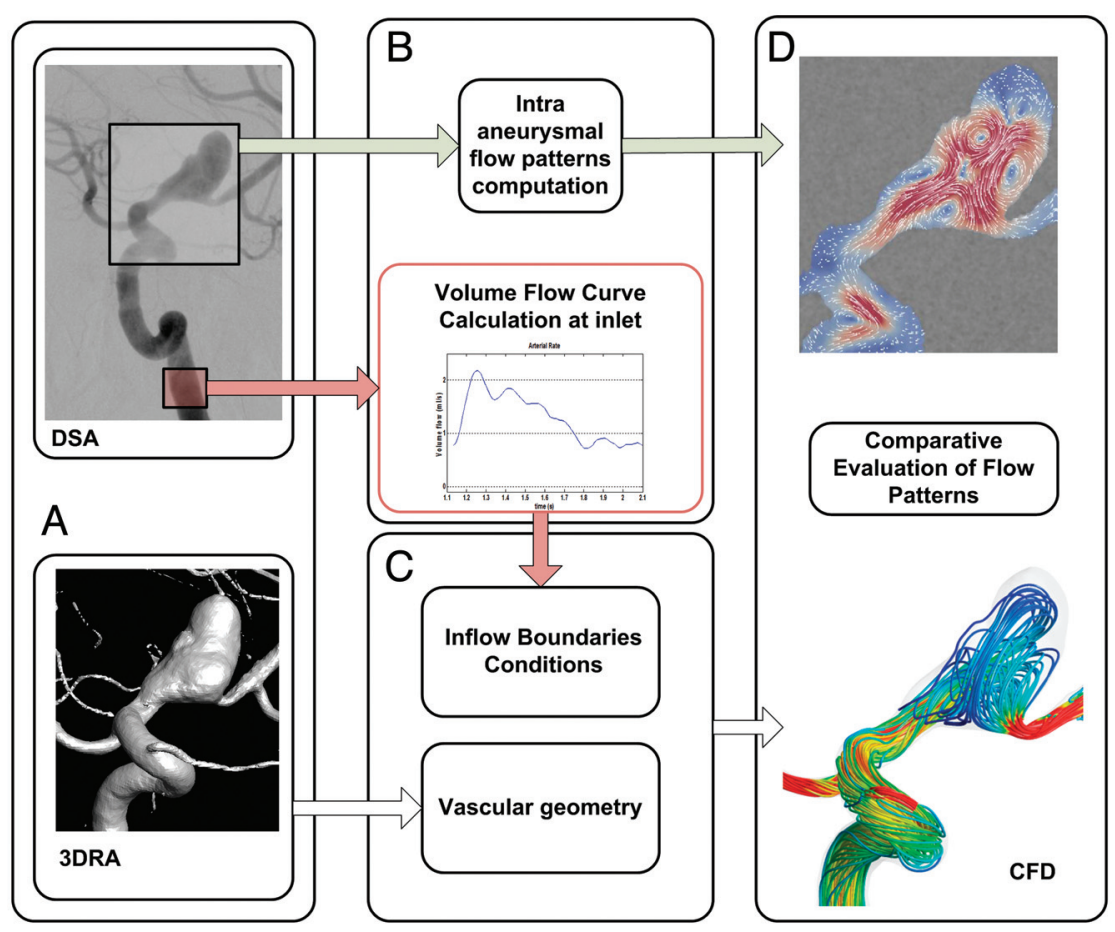

FIG 1. Workflow of the study. A, DSA and 3D rotational angiograms. B, Optical flow processing provides intra-aneurysmal flow patterns and volumetric flow curves measured in the parent vessel. C, Geometry and CFD modeling with the vessel geometry. D, Assessment of intra-aneurysmal flow patterns.

flow-diverter stent implantation and seems to be a good predictor of aneurysm thrombosis. ${ }^{6}$ The key success factors for this approach include low contrast-injection rates and an adaptive application of OF principles to high-frame-rate angiograms. These prerequisites allowed capturing the physiologic modulation of the contrast attenuation in blood flow, hence providing measurements of conebeam flat panel detector velocity fields (DVF). As a preliminary step ahead of OF in vivo validation in intracranial aneurysms, we propose to qualitatively compare the intra-aneurysmal flow patterns among DSA sequences (showing the qualitative gross flow structures), OF vectors in a $2 \mathrm{D}$ plane, and $3 \mathrm{D}$ streamlines based on computational fluid dynamics (CFD).

\section{MATERIALS AND METHODS}

\section{Patient Selection and Data Collection}

We included 21 consecutive patients who presented with unruptured saccular intracranial aneurysms before treatment. Patients with ruptured lesions, partially thrombosed aneurysms, or dissections were excluded from the study. This retrospective study was approved by the Geneva University Hospital ethics committee board (NEC 07-056).

\section{Data Collection}

Angiograms were performed by using the Seldinger technique at the level of the femoral artery. A 5F diagnostic catheter was used to selectively catheterize the vessel carrying the aneurysm. The catheter tip was positioned either in the internal carotid artery, $3 \mathrm{~cm}$ beyond the bifurcation, or at the V1 segment of the vertebral artery. A 3D rotational angiography was performed by using an angiographic biplane C-arm unit (Allura FD20; Philips Health- care, Best, the Netherlands) to provide the vascular geometries for CFD modeling and for the inlet volumetric blood flow OF measurement. ${ }^{5,6}$ Additionally, the $3 \mathrm{D}$ reconstruction was used to determine a suitable DSA projection view. The DSA projection view was chosen to ensure that overlapping the distal arterial branches and parent vessel from the aneurysm bulge was avoided to keep the region of interest free of irrelevant moving patterns that could affect measurements. Subsequently, a high-frame-rate DSA sequence (60 images/s) was acquired with a contrast agent injection rate of $1.5 \mathrm{~mL} / \mathrm{s}$ during 4 seconds for all patients.

\section{Optical Flow-Based Algorithm}

Using an OF method, ${ }^{4-6}$ we estimated the detector velocity fields on the basis of analyses of both the temporal and spatial variations of contrast agent attenuation injected during the DSA sequences. The temporal variations are spontaneously created when the injected contrast agent mixes with the blood stream under the pulsatile effect of the cardiac cycle while filling the vessel tree and the aneurysm. Bonnefous et al ${ }^{4}$ proposed to decouple the pulsating contrast attenuation from the nonmodulated wash-in/washout component. The contrast wave propagating through the vascular network was thereby captured and processed. The local temporal and spatial contrast attenuation variations were tracked among the successive images of the run. The result was a dynamic representation of DVF or streamlines that preserves their direction and magnitude. In addition, the vector fields were processed by imposing no divergent flow. Detailed descriptions of the postprocessing method can be found in Bonnefous et al ${ }^{4}$ and Pereira et al. ${ }^{6}$

\section{Vascular and CFD Modeling}

The triangulated surfaces of the vessels were first created from 3D rotational angiograms by using the AneuFuse toolkit (http:// www.biomedtown.org). ${ }^{7}$ Then, commercial software (ICEM CFD 12.1; ANSYS, Canonsburg, Pennsylvania) was used to produce a high-resolution computational unstructured mesh composed of tetrahedrons in the bulk flow and prism elements near the wall. The mesh element numbers ranged from 4.0 to 5.5 million, with a mesh attenuation of $>1700$ elements $/ \mathrm{mm}^{3}$. The computational mesh was used in the CFX commercial solver (CFX 12.1; ANSYS) to solve Navier-Stokes equations. Blood was assumed to exhibit the properties of a Newtonian incompressible fluid, and this was specified in the numeric solver. Inlet pulsatile boundary-condition flow curves were measured specifically for each patient by using the OF method coupled with the 3D rotational angiograms (Fig 1). ${ }^{4-6}$ These volumetric blood flow measurements were performed in the upstream parent vessel, far enough from the catheter tip to ensure good contrast agent mix- 


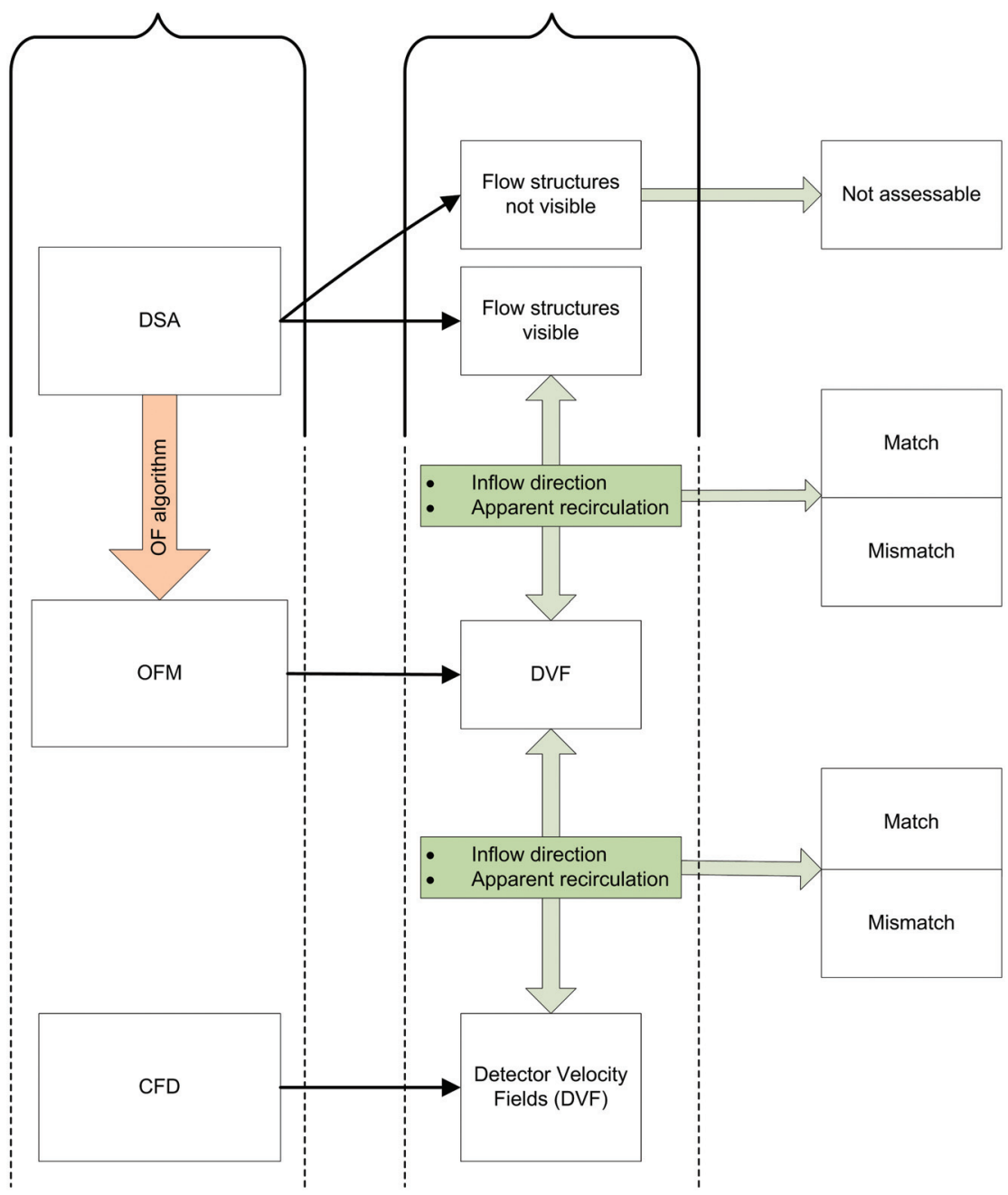

FIG 2. Comparative analysis among the 3 datasets: DSA, OF, and CFD images. The left column represents the 3 types of data work products initiating the illustrative analysis. The "evaluation" process is represented in the second column. The binary evaluation problems are represented in the last column for each defined element of the flow structures.

ing. ${ }^{4-6}$ The pressure boundary conditions of the outlets were based on measurements of healthy subjects, as published by Reymond et $\mathrm{al}^{8}{ }^{8}$ The Womersley analytic solutions, rescaled to the inlet diameter, were used to generate the specific distributions of velocity magnitudes over the artery diameter. The vessel wall was assumed rigid with no-slip boundary features. We simulated 2 cardiac cycles: The first cycle was used to reach solver convergence, and the second was used to collect the hemodynamic results for postprocessing. The Reynolds number ranged from 300 to 500, and the Womersley numbers, from 2.0 to 3.1. The analysis of the results was automated by using both commercial scripts from CFX and customized scripts from Matlab (MathWorks, Natick, Massachusetts).

\section{Comparative Evaluation of Flow Patterns}

All image datasets were displayed on the same view in the line of sight of the detector plane. The analysis workflow represented in Fig 2 compares 3 types of datasets:

- High-frame-rate DSA, in which gross intra-aneurysmal contrast motion structures were sought.

- OF images showing the extracted DVF outcomes: To facili- tate the visual comparison between DSA and OF images, we specifically integrated DVF to provide short streamlines starting and ending in the aneurysm region of interest (Fig 3, column 2).

- CFD results: The 3D streamlines were viewed with the camera aligned on the line of sight of the detector.

Datasets were visualized on a dedicated workstation and evaluated by 3 independent, experienced observers (V.M.P., O. Brina, and R.O.) blinded to each other's results; the interobserver evaluation agreement was then calculated by using Fleiss $\kappa$ statistics. For each patient, the 3 image sets were inspected at the systolic and diastolic phases and in the cine loops. Figure 3 shows a subset of flow patterns for the 3 datasets at systole to highlight the convective parts of contrast motion along with all the representation derivatives.

Because the vortical structures cannot be assessed on a planar detector, we restricted the comparative evaluation to inflow direction and apparent recirculation features (Table 1). The planar apparent circulation does not reflect the actual vortical structure on the detector. We evaluated the intra-aneurysmal flow features between the DSA and OF images and compared the DSA-OF images with the CFD outcomes.

\section{RESULTS}

Table 2 describes patient and aneurysm features of the 21 patients included in this study. Figure 3 summarizes the matching patterns of various degrees among the DSA, OF images, and CFD outcomes during the systolic phase, represented by 12 distinct aneurysm configurations and divided into 3 groups-Fig $3 \mathrm{~A}$ : conclusive with respect to DSA, DVF, and CFD outcomes ( 4 of 8 cases were represented); Fig $3 B$ : conclusive with respect to DVF and CFD outcomes (4 of 6 were represented); Fig 3C: inconclusive with respect to either DSA, DVF, or CFD outcomes (4 of 7 were represented). For all these cases, inlet flow was fed through the internal carotid artery or the vertebral artery. The flow rates ranged between 0.65 (a vertebral case) and $3.95 \mathrm{~mL} / \mathrm{s}$ (a carotid case).

\section{Comparison of DSA and OF Images}

Poor agreement was initially observed between DSA contrast motion patterns and OF velocity vectors because DSA was unable to provide any flow structure information in 13 of the 21 cases, despite the high-frame-rate resolution. These cases were classified as "nonassessable" and mainly involved small-sized aneurysms (Fig $3 B,-C)$. Alternatively, the patients presenting with larger lesions ( $>8 \mathrm{~mm}, n=8$ ) had well-defined DSA flow structures, all match- 


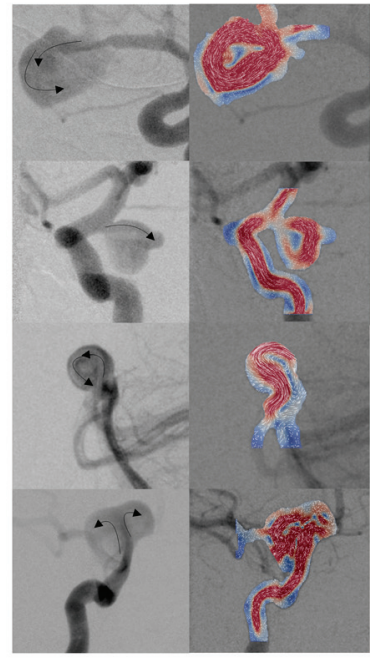

A
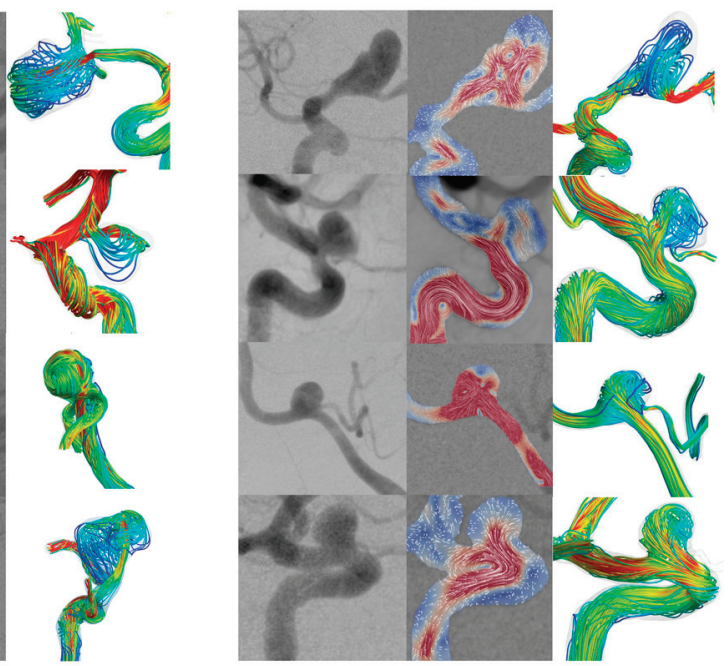

B

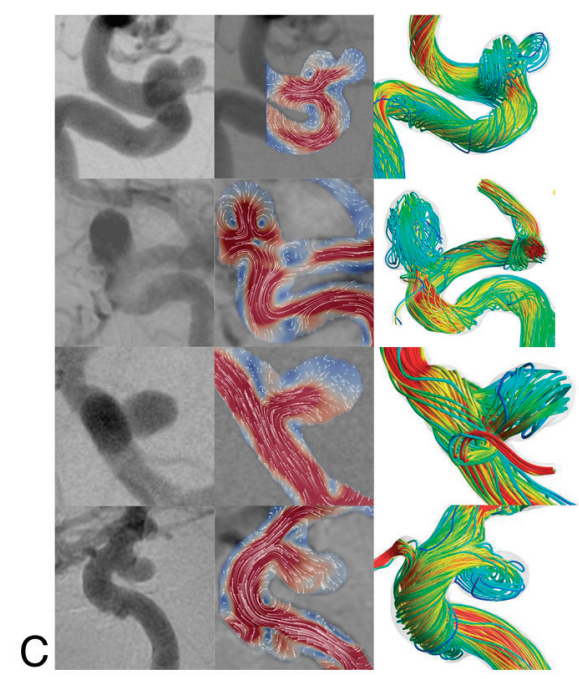

FIG 3. Illustration of the 3 classes of qualitative representations emanating from the 3 techniques: DSA, OF, and CFD. For each section ( $A-C$ ), the 3 columns from left to right represent DSA, DVF, and CFD images, respectively. Each row represents a different patient. Image captures correspond to the systolic phase. A, Four cases of 8 with flow patterns conclusively visible on DSA. The arrows show flow direction and apparent recirculation. The last 3 rows show perfect matching among DSA, OF images, and CFD, for instance, in patient 11 (row 2) shows a superior narrow inflow jet in a left posterior communicating artery aneurysm with a clockwise vortex. The first row represents patient 15 with a right middle cerebral artery aneurysm with consistent flow structures visible on both DSA and OF images (narrow jet entering the superior part of the aneurysm and creating an anticlockwise vortex). CFD streamlines show a different behavior represented by a medial jet, a narrow impingement area, and a planar vortex orthogonal to the DSA line of sight. This mismatch was essentially produced by fake stenosis generated by the vascular geometry modeling process. B, Four cases of 6 with a conclusive OF flow pattern, though "silent" (not visible) on DSA. For instance, DSA of patient 12 in row 3 shows no contrast motion in a left posterior inferior cerebellar artery aneurysm. Contrary to this finding, an inflow jet in the axis of the upstream vertebral artery was visible in the corresponding OF images. This trend was confirmed by CFD streamlines, though the jet deflection was incorrect. C, Four cases of 7 are inconclusive with either DSA, OF, or CFD images, all confirming that flow structures could not be visible on the selected plane of the detector. For instance, for the left carotid ophthalmic aneurysm seen in patient 21 and represented in row 2, the flow pattern was not visible in either the DSA, OF, or CFD images. Should the orthogonal projection view be chosen, the flow patterns would have been depicted better, as can be seen on the CFD image.

ing $\mathrm{OF}$ inflow direction and apparent recirculation representations (Fig $3 A$ ). Figure $3 A$, row 2, illustrates a perfect match between DSA and OF images, showing a superior narrow inflow jet along with a clockwise recirculation vortex in a posterior communicating artery aneurysm.
Additionally, even if DVF could be computed from DSA sequences for all the 21 patients, some cases presented $\mathrm{OF}$ patterns that could not be classified within the taxonomy of this study ( $n=$ 7) on the basis of inflow direction and apparent recirculation features. These cases all had "silent" DSA with no apparent flow structures (Fig 3C). Alternatively, Fig $3 B$ shows cases in which DSA was also inconclusive (no apparent flow structure), unlike in corresponding $\mathrm{OF}$ images $(n=6)$. All these evaluations were rated by 3 independent observers, with an average interobserver $\kappa$ estimated to 0.87 .

\section{Comparison of the OF Images and the CFD Outcomes}

In this step, we compared both inflow direction and apparent recirculation features from OF and CFD outcomes, taking into account the line of sight of the detector plane. The 14 cases that presented clearly defined OF patterns were confirmed by CFD, with the exception of 1 case illustrated in Fig $3 A$, row 1 . In this case, CFD streamlines matched neither DVF nor DSA images. This effect was caused by stenosis artifacts in the terminal part of the M1 segment generated by the segmentation process. This stenosis generated an inappropriate inflow jet in the medial part of the aneurysm, which was visible neither in DSA nor in DVF figures. In second row of Fig $3 A$, the strong CFD inflow jet coming through the neck was largely underestimated in OF images, even though its direction was consistent among the $3 \mathrm{da}$ tasets. Essentially, this underestimation was due to inappropriate temporal resolution in DSA. For 7 cases, DVF patterns could not be clearly defined (Fig 3C) and thereby compared with CFD outcomes. No mismatch was found between both techniques, while CFD streamlines were unable to characterizing DVF flow structures. The lack of OF image-pattern visibility in the line of sight of the detector as confirmed by CFD indicates that another DSA projection view would have been more suitable for flow-pattern visibility. For this OF-CFD comparison, the average interobserver $\kappa$ was 0.91 .

\section{DISCUSSION}

In this study, we investigated the qualitative degree of matching between OF-DVF and DSA in intracranial aneurysms with the 
Table 1: Inflow direction and apparent recirculation features

\begin{tabular}{ccc}
\hline Parameters & Description & Label \\
\hline Inflow direction & Intra-aneurysmal orientation of & Match nonmatch \\
& the penetrating flow/jet & \\
$\begin{array}{cc}\text { Apparent } & \text { Presence of vortical structures/ } \\
\text { recirculation } & \text { vortex rotation direction }\end{array}$ \\
\hline
\end{tabular}

Table 2: Summary of clinical information ${ }^{a}$

\begin{tabular}{|c|c|c|c|c|c|c|c|}
\hline Patient & Sex & $\begin{array}{l}\text { Age } \\
\text { (yr) }\end{array}$ & $\begin{array}{c}\text { Aneurysm } \\
\text { Location }\end{array}$ & $\begin{array}{c}\text { Size H/L/W } \\
(\mathrm{mm})\end{array}$ & $\begin{array}{c}\text { Neck } \\
\text { Length } \\
(\mathrm{mm})\end{array}$ & $\begin{array}{c}\text { Volume } \\
\left(\mathrm{cm}^{3}\right)\end{array}$ & AR \\
\hline 1 & $\mathrm{~F}$ & 70 & LMCA & $4.6 / 4.8 / 4.7$ & 3.90 & 0.076 & 1.2 \\
\hline 2 & $\mathrm{~F}$ & 54 & RMCA & 8/10.6/8.3 & 9.90 & 0.48 & 0.8 \\
\hline 3 & $F$ & 60 & BaTip & 6.1/7.1/6.7 & 5.10 & 0.2 & 1.18 \\
\hline 4 & $\mathrm{~F}$ & 60 & $\mathrm{RCO}$ & $4.9 / 8.7 / 5.3$ & 4.60 & 0.122 & 1 \\
\hline 5 & $\mathrm{~F}$ & 58 & LCO & $6.2 / 6.2 / 4.7$ & 3.80 & 0.124 & 1.6 \\
\hline 6 & M & 62 & LMCA & $12.4 / 8.4 / 6.8$ & 10.40 & 0.64 & 1.2 \\
\hline 7 & $\mathrm{~F}$ & 62 & $\mathrm{RCO}$ & $6.6 / 5.6 / 5.8$ & 3.90 & 0.115 & 1.7 \\
\hline 8 & M & 50 & LICA cave & $4 / 4.5 / 6$ & 4.20 & 0.066 & 0.95 \\
\hline 9 & $\mathrm{~F}$ & 67 & LPcomA & 6.9/7.7.10.8 & 7.80 & 0.47 & 0.9 \\
\hline 10 & M & 40 & RICA cave & $3.8 / 4.3 / 4.2$ & 4.70 & 0.082 & 0.8 \\
\hline 11 & $\mathrm{~F}$ & 47 & LPcomA & $9.3 / 9.1 / 6.2$ & 2.25 & 0.22 & 4.15 \\
\hline 12 & $\mathrm{~F}$ & 55 & LPICA & $6.6 / 5.2 / 6.6$ & 6.10 & 0.19 & 0.85 \\
\hline 13 & $\mathrm{~F}$ & 50 & RICA & $13.5 / 17.8 / 9.9$ & 7.60 & 1.13 & 1.77 \\
\hline 14 & $\mathrm{~F}$ & 53 & LCO & $5.3 / 5.5$ & 4.40 & 0.111 & 1.2 \\
\hline 15 & $\mathrm{~F}$ & 54 & RMCA & 18.3/14.9/13.85 & 8.50 & 2.3 & 2.15 \\
\hline 16 & $\mathrm{~F}$ & 56 & RICA & $4.2 / 5.6 / 4.5$ & 3.80 & 0.069 & 0.9 \\
\hline 17 & $F$ & 45 & LCO & $5.9 / 7.7 / 6.7$ & 3.80 & 0.173 & 1.3 \\
\hline 18 & $\mathrm{~F}$ & 64 & RICA & $18.3 / 17 / 18.5$ & 8.50 & 3.125 & 2.2 \\
\hline 19 & $\mathrm{~F}$ & 56 & LICA & $3.4 / 3.2 / 3$ & 2.90 & 0.026 & 1 \\
\hline 20 & $\mathrm{~F}$ & 56 & LCO & $5 / 5.1 / 5.9$ & 4.50 & 0.109 & 1 \\
\hline 21 & $\mathrm{~F}$ & 52 & LCO & $8.2 / 9.6 / 8.5$ & 4.60 & 0.353 & 1.7 \\
\hline
\end{tabular}

Note:-AR indicates aspect ratio; BaTip, basilar artery tip; $\mathrm{CO}$, carotid-ophthalmic; PcomA, posterior communicating artery; PICA, posterior inferior cerebellar artery; L, left; $\mathrm{R}$, right; $\mathrm{H} / \mathrm{L} / \mathrm{W}$, height/length/width.

${ }^{a}$ Columns represent data for each patient.

help of CFD. The 3 techniques varied greatly in terms of the physical property that they addressed: Digital subtraction angiography essentially measures the absorption of $\mathrm{x}$-rays and contrast motion during the cardiac cycle, the optical flow technique extracts the physical modulation of contrast attenuation over the cardiac cycle out of which in-plane-projected velocity fields (DVF) are assessed, and CFD calculates the 3D velocity field of blood flow by using the inlet boundary conditions provided by both DSA sequences and $3 \mathrm{D}$ images. These techniques have been used jointly to improve our interpretation of OF outcomes, which were successfully used to predict flow-diverter stent treatment issues in intracranial aneurysms. ${ }^{6}$ Although the OF method has allowed matching between contrast motion and convective flow, the observation of genuine or fake flow structures generally depends on the complexity of flow and the direction of detector plane. Therefore, CFD-computed streamlines were needed to dissipate the above-mentioned ambiguities to avoid any misleading interpretation.

In particular, it was first shown that DVF figures obtained from DSA fairly matched CFD outcomes for both the inflow direction and the apparent recirculation. The complexity of the flow structure, which could be reliably represented by CFD streamlines over the cardiac cycle, helped greatly in drawing conclusive evaluations in the chosen plane of the detector. In our study, 7 of 21 cases were not conclusive as was expected from DSA figures in out-of-focus projection planes. Of the 14 conclusive OF flow structures, only 8 were conclusive with regard to DSA images. The 6 others were not. Nevertheless, their representations of inflow direction and apparent recirculation were compatible with CFD streamlines, independent of aneurysm size, thereby demonstrating that the OF method could possibly unfold genuine flow structures wherever hidden in DSA. The qualitative analysis among DSA, OF images, and CFD was in perfect agreement among the 3 independent observers, as demonstrated by the high $\kappa$ values of $>0.8$, confirming that based on the described binary parameters, OF images can provide consistent flow information. Unlike in videos, still images did not necessarily reflect the degree of agreement among the observers.

The clinical benefits of these DVF measurements, readily available during treatment, have not yet been fully established. However, one can foresee several applications in the future: 1) predicting thrombosis in stented aneurysms, ${ }^{6}$ and 2) adapting CFD to perioperative patient-specific conditions. Furthermore, an extension of the OF method in 3D would help give more quantitative insight on both the measurements of flow-pattern complexity and related parameters such as wall shear stress in aneurysms, which are believed to play an important role in all aspects of aneurysm growth, ${ }^{9}$ rupture, ${ }^{10}$ and clotting. ${ }^{11}$

In line with Taylor and Steinman, ${ }^{12}$ the OF method was based on vascular anatomic imaging criterion standard DSA, which could bring flow evaluation closer to daily clinical practice. Taylor and Steinman strongly encouraged bringing image-based modeling to the management of vascular diseases rather than innovating with more sophisticated developments that diverge from the clinically oriented approaches. Alternatively, other groups have followed the same lead by using phase-contrast MR angiography and CFD comparisons. Karmonik et $\mathrm{al}^{13}$ described similar velocity shapes between 2D phase-contrast MR angiography and CFD in anterior communicating artery aneurysms. In 2 canine aneurysm models, Jiang et al $^{14}$ also showed favorable agreement of velocity fields between phase-contrast MR angiography and CFD. Other studies compared the flow patterns between DSA and CFD by using virtual angiogram modeling. Cebral et $\mathrm{al}^{15}$ qualitatively compared the intra-aneurysmal flow structures of 3 patients between conventional DSA and virtual angiograms and were able to predict, with good agreement, the locations and sizes of the inflow jet, outflow, impaction zone, and vortex structures.

\section{Limitations and Further Developments}

The single DSA projection view is an unfortunate limitation of our method, which restricted the CFD analysis and consequently precluded the use of qualitative features defined, for instance, by Cebral et $\mathrm{al}^{10}$ (inflow concentration, impingement zone, flow complexity, and flow stability) to describe the intra-aneurysmal flow. These features were not fully applicable in the context of our DVF study implying the use of simpler parameters. Because the optical flow algorithm measures moving patterns within the regions of interest, overlap of branches and the parent vessel should be avoided as much as possible when choosing the projection views. Moreover, the OF method might introduce fake vortices as a result of projecting laminar flow patterns, which can only be avoided by the extension of the method to 3D images. DVF mag- 
nitude can be underestimated for very high velocities due to inappropriate temporal resolution in DSA. In addition to optical flow method boundary condition measurements, segmentation may also impact CFD outcome as has been observed in the first patient in Fig 3A. Furthermore, both the seed points and attenuation may influence CFD streamlines.

Moreover, even if the OF method was validated by using Doppler sonography to evaluate the volumetric blood flow in internal carotid arteries, we believe that intra-aneurysmal flow quantification in patients would require further development and carefully conducted validation steps involving, for example, particle imaging velocimetry measurements. In this study, we aimed to demonstrate that a novel method, applicable to the clinical routine, could be used in the future to perform more patientspecific CFD and to characterize potential aneurysm flow patterns related to rupture.

\section{CONCLUSIONS}

Velocity field patterns measured in intracranial aneurysms by using the OF method from DSA sequences were reasonably matched with DSA and CFD outcome and could provide, whenever possible, complementary flow pattern structures not visible on DSA. Moreover, the extension of the OF method to 3D images should definitely help in removing fake single-plane flow structures.

Disclosures: Karl Schaller-UNRELATED: Grants/Grants Pending: Swiss National Science Foundation. ${ }^{*}$ Vitor Mendes Pereira—RELATED: Grant: Philips, ${ }^{*}$ Comments: The grant is to pay the $50 \%$ time of a research technician who works locally in our center to collect the data and work on the postprocessing for our daily clinical practice and for the related research projects that we have with Philips. *Money paid to the institution.

\section{REFERENCES}

1. Vlak MH, Algra A, Brandenburg R, et al. Prevalence of unruptured intracranial aneurysms, with emphasis on sex, age, comorbidity, country, and time period: a systematic review and meta-analysis. Lancet Neurol 2011;10:626-36

2. Pereira VM, Brina O, Bijlenga $P$, et al. Wall shear stress distribution of small aneurysms prone to rupture: a case-control study. Stroke 2014;45:261-64
3. Pereira VM, Brina O, Gonzalez AM, et al. Biology and hemodynamics of aneurismal vasculopathies. Eur J Radiol 2013;82:1606-17

4. Bonnefous OP, Pereira VM, Ouared R, et al. Quantification of arterial flow with digital subtracted angiography (DSA). Med Phys 2012;39:6264-75

5. Pereira VM, Ouared R, Brina O, et al. Quantification of internal carotid artery flow with digital subtraction angiography: validation of an optical flow approach with Doppler ultrasound. AJNR Am J Neuroradiol 2014;35:156-63

6. Pereira VM, Bonnefous O, Ouared R, et al. A DSA-based method using contrast-motion estimation for the assessment of the intraaneurysmal flow changes induced by flow-diverter stents. AJNR Am J Neuroradiol 2013;34:808-15

7. Villa-Uriol MC, Berti G, Hose DR, et al. @neurIST complex information processing toolchain for the integrated management of cerebral aneurysms. Interface Focus 2011;1:308-19

8. Reymond P, Merenda F, Perren F, et al. Validation of a one-dimensional model of the systemic arterial tree. Am J Physiol Heart Circ Physiol 2009;297:H208-22

9. Boussel L, Rayz V, McCulloch C, et al. Aneurysm growth occurs at region of low wall shear stress: patient-specific correlation of hemodynamics and growth in a longitudinal study. Stroke 2008;39: 2997-3002

10. Cebral JR, Mut F, Weir J, et al. Association of hemodynamic characteristics and cerebral aneurysm rupture. AJNR Am J Neuroradiol 2011;32:264-70

11. Ouared R, Chopard B, Rufenacht D, et al. Thrombosis Engineering in Intracranial Aneurysms Using a Lattice Boltzmann Numerical Method. Munich: Springer-Verlag; 2009:1538-41

12. Taylor CA, Steinman DA. Image-based modeling of blood flow and vessel wall dynamics: applications, methods and future directions-Sixth International Bio-Fluid Mechanics Symposium and Workshop, March 28-30, 2008 Pasadena, California. Ann Biomed Eng 2010;38:1188-203

13. Karmonik C, Klucznik R, Benndorf G. Comparison of velocity patterns in an AComA aneurysm measured with 2D phase contrast MRI and simulated with CFD. Technol Health Care 2008;16:119-28

14. Jiang J, Johnson K, Valen-Sendstad K, et al. Flow characteristics in a canine aneurysm model: a comparison of $4 \mathrm{D}$ accelerated phasecontrast MR measurements and computational fluid dynamics simulations. Med Phys 2011;38:6300-12

15. Cebral JR, Pergolizzi RS Jr, Putman CM. Computational fluid dynamics modeling of intracranial aneurysms: qualitative comparison with cerebral angiography. Acad Radiol 2007;14:804-13 